\title{
Matching characteristics of propeller and engine of fuel-powered quadrotor aircraft
}

\author{
Fupei Zheng ${ }^{1,2, a^{*}}$, Weijun Wang ${ }^{1, b}$, Song $\mathrm{Li}^{3, \mathrm{c}}$ and Jicheng Zhang ${ }^{3, \mathrm{~d}}$ \\ ${ }^{1}$ School of Aeronautical Science and engineering, Beihang University, Beijing,China \\ ${ }^{2}$ Air Force XI an Flight Academy, Xian,China \\ ${ }^{3}$ Air Force Aviation University, Jilin, China \\ az-f-p@126.com, bweijun-w@126.com, clisong4885@126.com, ${ }^{\mathrm{d}}$ zhangjc@126.com
}

Keywords: fuel-powered quadrotor aircraft; rotor CFD; match of propeller and engine.

Abstract. Quadrotor aircraft has become the forefront and hotspot in the research of the aviation industry. At present, the study of the quadrotor aircraft mainly concentrated in the category of electric quadrotor aircraft. But the electric quadrotor aircraft has a relatively short range and small payload, mainly restricted by battery capacity. In order to improve its shortage, select fuel engine as a driving force of the quadrotor aircraft and variable pitch propeller as its propeller. Using computer CAD technology to establish the modeling of different diameter and pitch propeller, then classify the structural grid, and finally with the help of fluid mechanics computing platforms to simulate the propeller's hover state of different diameter and pitch. Matching characteristics analysis of different calculation results with engine performance parameters was carried and conclusion was drawn about the matching law of fuel-powered quadrotor aircraft propeller and engine. This work can provide reference for the overall design of fuel-powered quadrotor aircraft.

\section{Introduction}

Quadrotor aircraft now belongs to the category of the unmanned aerial vehicle (UAV). It is a kind of un-coaxial rotor multi-rotor aircraft and has vertical take-off and landing performance, as well as has a low cost. Now, it can pick up certain of the aviation equipment to fly by itself and has a broad application prospect in the fields of buffet for disaster relief, field investigation, monitoring and prevention and cure of diseases and pests of agriculture and forestry, film and television entertainment, scientific research and so on.

Nowadays, more common quadrotor aircraft is micro-electric quadrotor aircraft such as Draganflyer X4 by American Draganflyer Company ${ }^{[1]}$ and X-UFO ${ }^{[2]}$ by Hong Kong silver Company. Both of these two are remote control model aircraft. In terms of Micro Aerial Vehicle, one of them is the famous Mesicopter miniature rotor aircraft ${ }^{[3]}$, which can realize autonomous flight and multi-aircraft flight together to complete the task.

In recent years, researches on quadrotor aircraft mainly focused on the control algorithm and its stability. The representative algorithms include OS4 developed by Swiss federal institute of technology in lausanne (EPFL) ${ }^{[4]}$. Until 2004, flight attitude control has been achieved using a variety of algorithm PID ${ }^{[5]}$, Back stepping ${ }^{[6]}, \mathrm{LQ}^{[7]}$ and Sliding - mode ${ }^{[8]}$ based on OS4. In addition, algorithm HMX4 developed by the university of Pennsylvania ${ }^{[7]}$ is also used widely, which is mainly based on visual autonomous flight control. In domestic, many universities are carrying on researches on quadrotor aircrafts ${ }^{[9]}$ such as tsinghua university, Shanghai Jiaotong University ${ }^{[10]}$, national defense science and technology university ${ }^{[8]}$, Beihang University and Nanjing university of aeronautics and astronautics ${ }^{[11]}$, etc.

With the deepening of the quadrotor aircraft applications, micro electric quadrotor aircraft appeared some limitations, such as short endurance and small flight radius, etc., so the fuel-powered quadrotor aircraft occured. Because fuel engine has much larger power than air motor, and is not limited by battery storage capacity, the more power greatly increases the payload of the aircraft and fuel load, so the range and endurance increased. Therefore, the research about fuel-powered quadrotor aircraft is of much more value. Propeller and engine are both the main parts of the 
quadrotor. If they are matched well, the aircraft will perform greatly. So the matching problem between them is an important topic. two important geometric parameters influencing aerodynamic performance of propeller are diameter and pitch. In this paper, propeller models with different diameter and pitch are established and their flow fields of CFD are computed, respectively. Analyzing the results based on the selected engine model, we can finally conclude the corresponding matching rules, which will provides a theoritical reference for the overall design of fuel-powered quadrotor aircraft.

\section{Establishment of Calculation Model.}

Calculation model mainly includes the choice of engine model, the determination of propeller model and the computational domain. Establishment on engine model is focused on engine performance data available and propeller model is to design and calculation the geometry size of propeller. With the aid of computer CAD technology, envelope the propeller model into entities, and develop different propeller model on the basis of the basic propeller model. Determination of computational domain is mainly to determine the size of the peripheral flow field, which usually relys on experience to determine.

Engine Model. Select Rotax912S as the engine model. Its basic parameters is shown in table 1. The working time limit under the condition of the largest state is $5 \mathrm{~min}$. Due to the normal working condition is in its rated condition, so the rated condition is the normal maximum state, which is the state of the propeller matching to.

Table 1 Basic Parameters Rotax912S Engine

\begin{tabular}{|l|l|}
\hline Model & Rotax912S \\
\hline Rated speed & $71.38[\mathrm{kw}], 5500[\mathrm{rpm}]$ \\
\hline Maximum speed & $73.5[\mathrm{kw}], 5800[\mathrm{rpm}]$ \\
\hline $\begin{array}{l}\text { Specific fuel } \\
\text { consumption }\end{array}$ & $194[\mathrm{~g} / \mathrm{hp} \cdot \mathrm{h}]$ \\
\hline Weight & $56.6[\mathrm{~kg}]$ \\
\hline
\end{tabular}

Propeller Model. First, establish a baseline propeller of size $1 \mathrm{~m}$ (39.37 inch). Its pitch is 406.4 $\mathrm{mm}$ (16 inch), named as P_3916. The airfoil of it is CLARK Y. the thickness of the airfoil is $12 \%$ of the chord length. The design of the benchmark position depends on the general rules. The following other designs of the propeller model is conducted according to this benchmark and scaling. The geometry data of the propeller P_3916 model is shown in table 2 and the $\mathrm{P} \_3916$ propeller model is shown in figure 1. Applying the parametric design to establish the model, then different propeller model with different diameter and pitch can come out on the basis of the benchmark model promotion.

Determination of Calculation Domain. Because the propeller is a rotating parts, choose a cylinder acts as the calculation domain of propeller and wrap the propeller in it. In this article, the radius of the computational domain is 10 times of the cylinder's radius, the upper height of the propeller is 10 times of the cylinder's radius and the bottom height is the twice of the upper one. $\mathrm{R}$ here refers to the radius of benchmark propeller model, which is $500 \mathrm{~mm}$. For P_3916 propeller, the radial diameter of circumferential calculation domain is $10000 \mathrm{~mm}$, the distance to the upper is 5000 $\mathrm{mm}$ and the distance to the bottom is $10000 \mathrm{~mm}$. There are two boundaries and one area. The outer boundary is the external cylindrical surface. In the CFD calculation, it is set as the far field boundary, that is the pressure-far-field, which is the boundary of the air is not disturbed. The internal boundary is the surface of the propeller model, which is set as wall. The area between the inside and outside the boundary is the fluid field, and is set as fluid. 
Table 2 Geometry Data of Benchmark Propeller Model

\begin{tabular}{|c|c|c|c|c|c|c|c|}
\hline $\begin{array}{c}\text { Radius } \\
{[\mathbf{m m}]}\end{array}$ & Blade Angle & $\begin{array}{c}\text { Length of } \\
\text { Chord }\end{array}$ & $\begin{array}{c}\text { Ratio of } \\
\text { Thickness }\end{array}$ & $\begin{array}{c}\text { Thickness } \\
{[\mathbf{m m}]}\end{array}$ & $\begin{array}{c}\text { Lay-out } \\
\text { of Blade }\end{array}$ & $\begin{array}{c}\text { Ratio } \\
\text { of Y } \\
\text { Axis }\end{array}$ & $\begin{array}{c}\text { Ratio of } \\
\text { Z axis }\end{array}$ \\
\hline 50 & 0.912718397 & 50 & $50 \%$ & 25 & $10 \%$ & 0.5 & 2.083333 \\
\hline 100 & 0.57412638 & 78.12 & $30 \%$ & 23.436 & $20 \%$ & 0.7812 & 1.953 \\
\hline 150 & 0.407113559 & 94.677 & $18 \%$ & 17.04186 & $30 \%$ & 0.94677 & 1.420155 \\
\hline 200 & 0.312786649 & 100 & $12 \%$ & 12 & $40 \%$ & 1 & 1 \\
\hline 250 & 0.253170867 & 98.62 & $12 \%$ & 11.8344 & $50 \%$ & 0.9862 & 0.9862 \\
\hline 300 & 0.212351392 & 94.479 & $12 \%$ & 11.33748 & $60 \%$ & 0.94479 & 0.94479 \\
\hline 350 & 0.182739949 & 87.568 & $12 \%$ & 10.50816 & $70 \%$ & 0.87568 & 0.87568 \\
\hline 400 & 0.160313773 & 77.863 & $12 \%$ & 9.34356 & $80 \%$ & 0.77863 & 0.77863 \\
\hline 450 & 0.142756854 & 65.352 & $12 \%$ & 7.84224 & $90 \%$ & 0.65352 & 0.65352 \\
\hline 500 & 0.128646708 & 50 & $12 \%$ & 6 & $100 \%$ & 0.5 & 0.5 \\
\hline \multicolumn{7}{|c|}{ Diameter of Hub: $160[\mathrm{~mm}]$ Height of Upper.60 [mm] Height of Bottom: $50[\mathrm{~mm}]$} \\
\hline
\end{tabular}

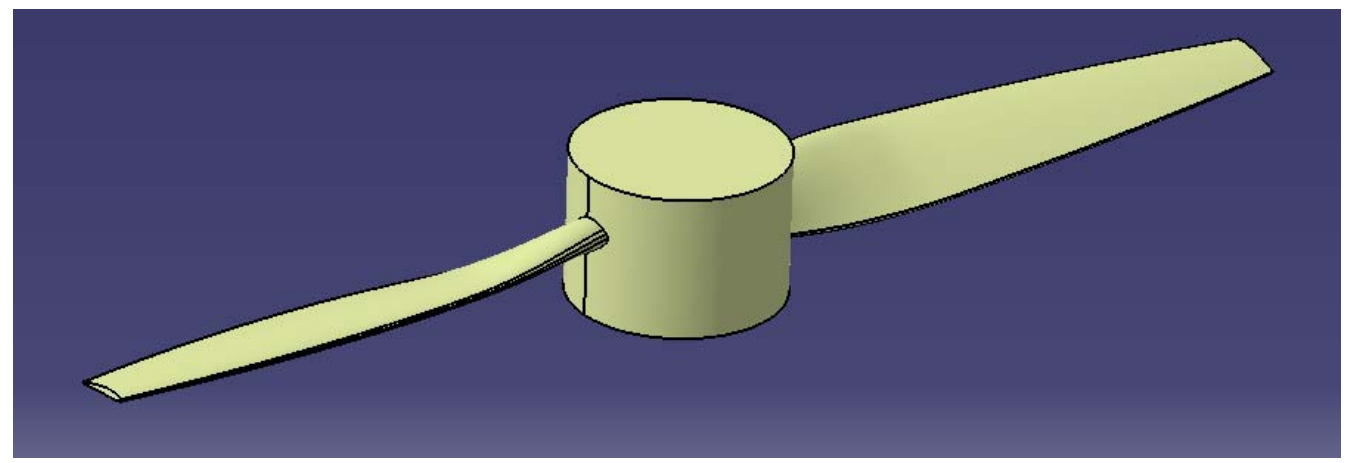

Fig. 1 Benchmark model of propeller

Structural Grid Meshing of Propeller's Calculation Domain. It is a difficulty to mesh the propeller according to the structure grid, because it is twisted along the span of the blade. For this kind of complex geometric surface, unstructured grid is relatively easy as shown in figure 2 . But structural grid has many advantages such as less resource-intensive, faster computing speed and better convergence and higher accuracy. It has attracted many people to research and explore. As shown in figure 3, it is the grid structure local map of propeller P_3916.

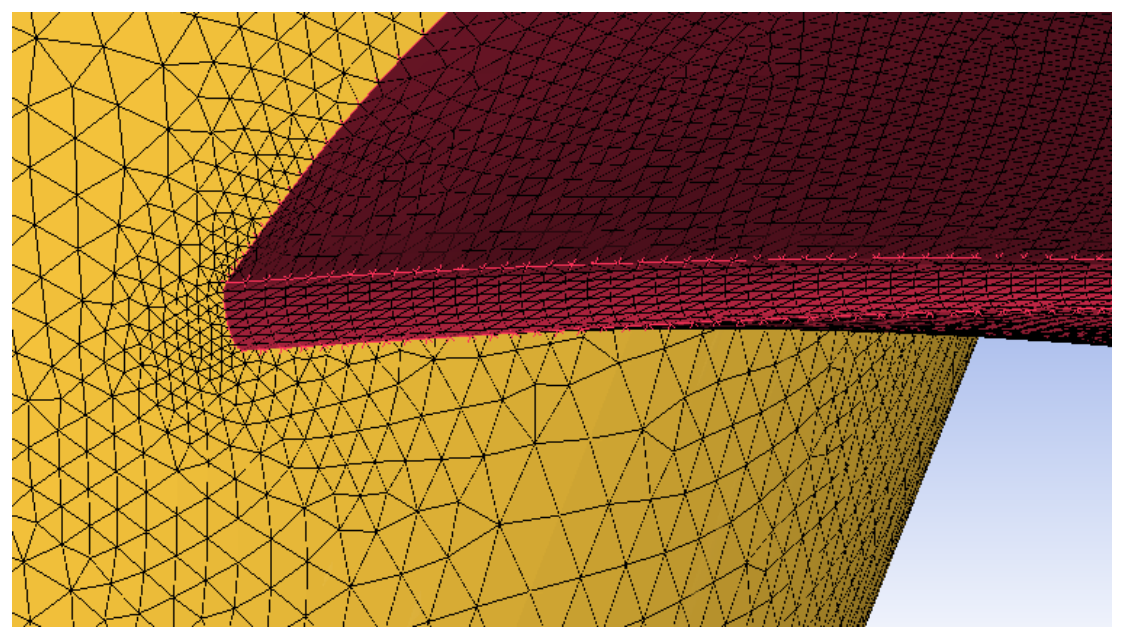

Fig. 2 Unstructured grid of propeller 


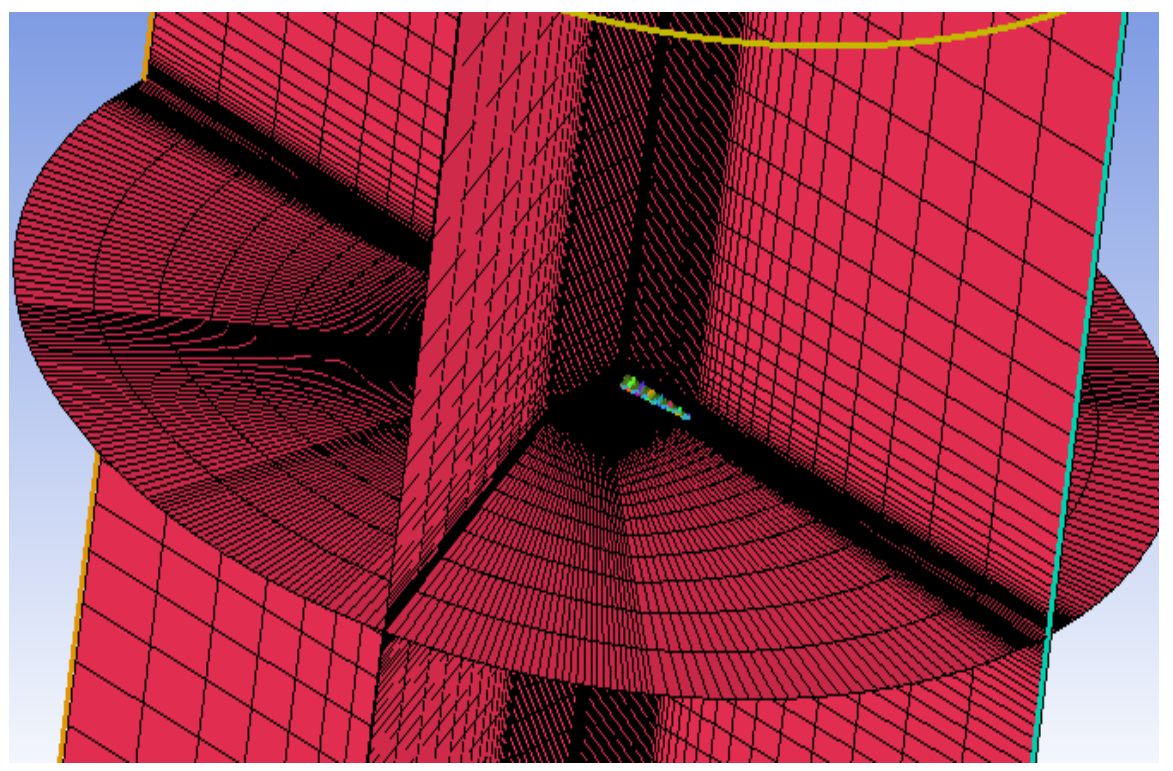

Fig. 3 Structured grid of propeller

\section{Matching Analysis between the Propeller and Engine}

By CFD calculation of propeller aerodynamic parameters including hovering under different rotational speed when the input power, torque, such as size to match it to the engine rated working condition. Finally be rated tensile strength of the state of the propeller, the input parameters such as power, torque and transmission ratio, tip Mach number, on the basis of these parameters to evaluate the stand or fall of matching.

Matching Model. This paper involves fuel-powered quadrotor aircraft is four main rotor, driven by a motor on the regulation law adopts constant engine speed. The constant speed is its rated speed $5500 \mathrm{rpm}$, the transmission efficiency is supposed as $90 \%$, the input power from the engine rated power is converted into a propeller. Four propeller speed equal to the rated speed of engine. There is a problem of ratio. When it is in hovering, four propeller distribute the total power of the engine averagely, so it simplifies the problem. As long as the calculation of a propeller parameters, the related parameters conversion into four general parameters of the propeller.

Example. Take P_3916 as an example to study the matching characteristics under the engine rated condition matching. The CFD calculation results are shown in figure 4.

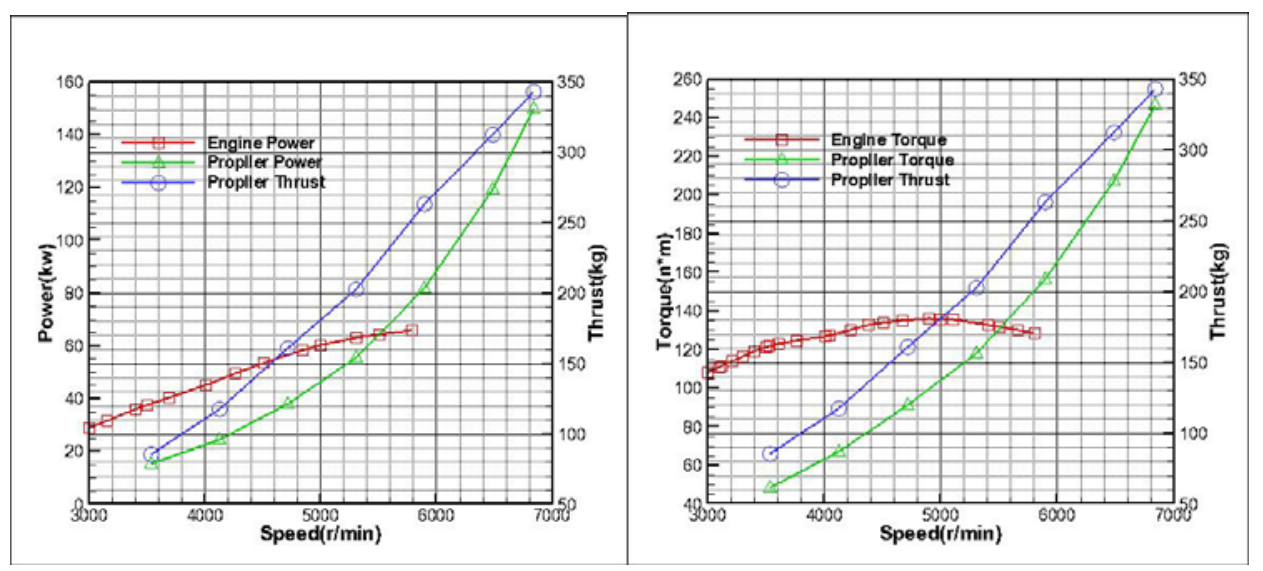

Fig. 4 Matching curve of propeller and engine of $P$ _3916 
It can be seen from figure 4, both the power and the torque of propeller match the rated condition of engine. At this time, the power and torque is equal to the intersection point, which is corresponding to the rated speed of engine $5500 \mathrm{r} / \mathrm{min}$.

Best Match of the Propeller. In this work, 79 propeller CFD calculations were conducted, mainly by the change of the diameter and pitch to match. Comparisons between different propellers with different diameter and same pitch (16 inch) are shown in figure 5acomparisons between propeller with the same diameter (46 inch) and different pitch are shown in figure 6 . Finally for the propeller under different diameter calculate pitch. The results show that the regularity of its change is similar to the characterization of figure 5. In these two cases, you can find the optimal pitch corresponding to the maximize pitch. Figure 7 illustrates the match rule in charting form.

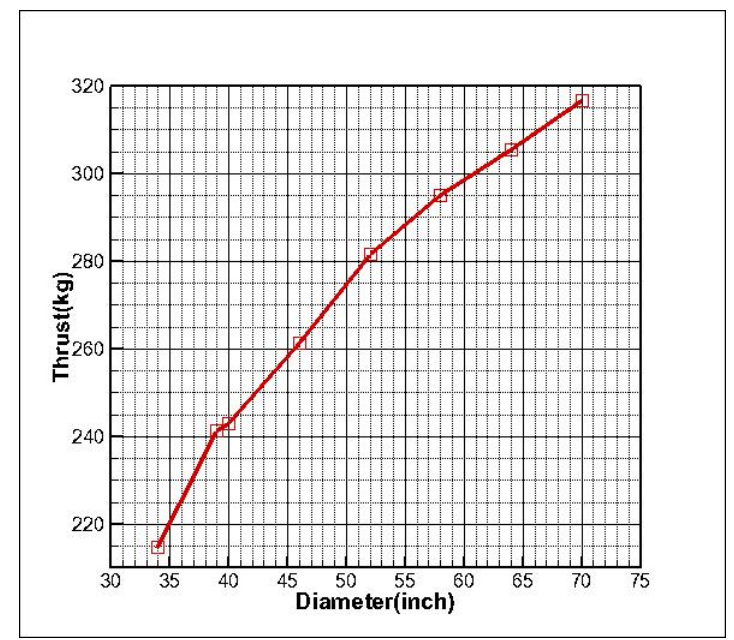

Fig. 5 Comparison of propeller's pull with different diameter

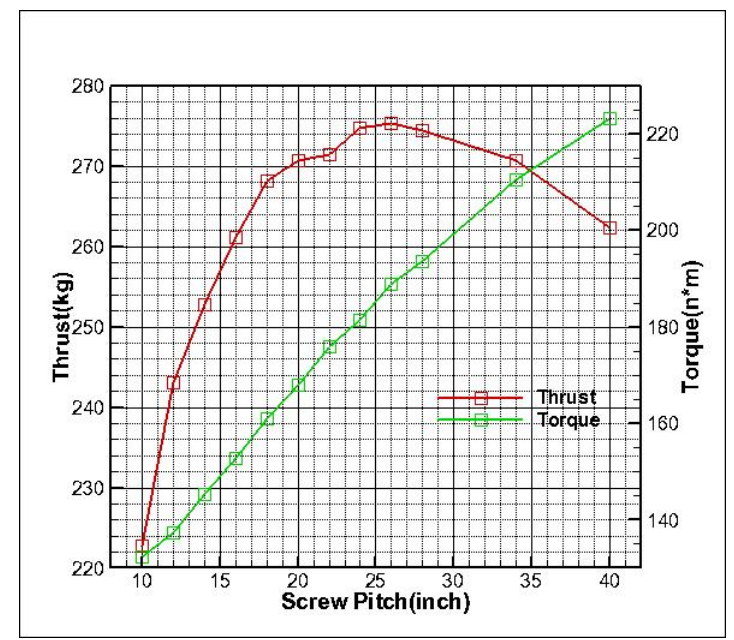

Fig. 6 Influence of pitch on the matching characteristics

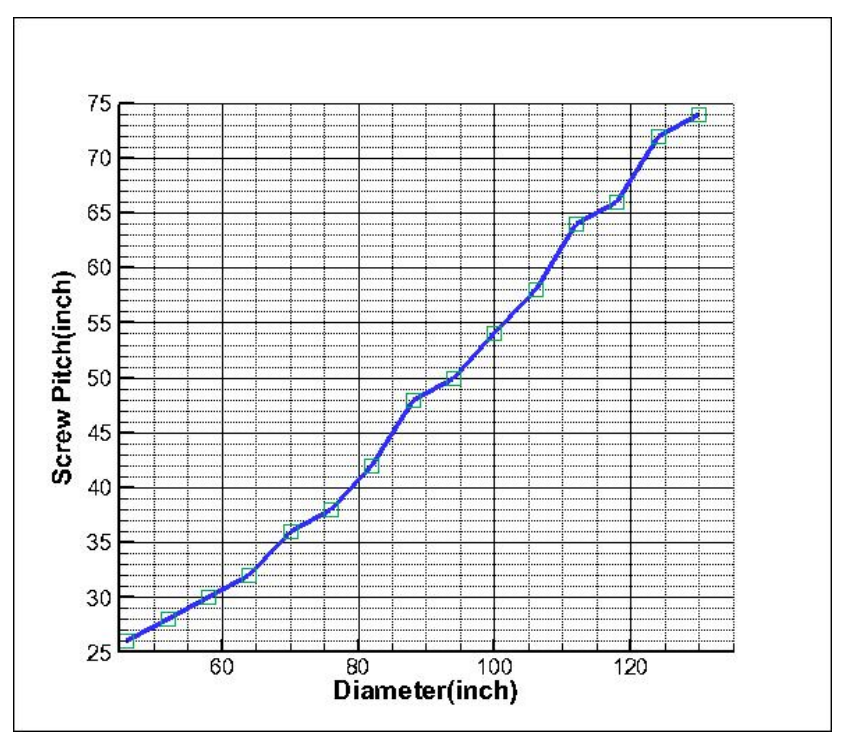

Fig. 7 Best matching curve of propeller and engine

\section{Conclusion}

1. Numerical simulation of matching between propeller and engine of fuel-powered quadrotor aircraft can be carried on using CFD calculation domain model, and the best parameters of the propeller can be determined. 
2. For the selected engine, on the premise of the structure and strength allowed, the larger the matching propeller diameter is, the greater the pull provided by the propeller.

3. For the propeller with given diameter, there must be a best matching pitch to the selected engine.

4. when the engine is given, there is a trend of large propeller blade with a large pitch, and the small blade with a small one for the matching propeller.

\section{References}

[1] Mckerrow P. Modelling the Draganflyer four-rotor helicopter[C]// Faculty of Informatics Papers. 4(2004)3596-3601.

[2] Bowen Nie, Hongxu Ma, Jian Wang, etc. The research status and key technology of micro four-rotor aircraft [J]. Electronics Optics \& Control, 14(2008)113-117.

[3] Zhanke Li, Bifeng Song, Hailong Song. The research status and key technology of MAV [J]. Journal of flight mechanics, 21 (2004) 1-4.

[4] Bouabdallah S, Siegwart R.Towards intelligent miniature flying robots[C]//Proceedings of Field and Service Robotics, Australia,Port Douglas, 2005.

[5] Bouabdallah S, Noth A,Siegwart R. PID vs LQ control techniques applied to an indoormicro-quadrotor[C]/Proceedings of the IEEE International Conference on Intelligent Robots and Systems(IROS),(2004)2451-2456.

[6] Bouabdallahs S, Siegart R. Backstepping and sliding-mode techniques applied to an indoor micro quadrotor[C]/Proceedings of International Conference on Robotics and Automation,Barcelona,2005.

[7] Altug E. Vision based control of unmanned aerial vehicles with applications to autonomous four rotor helicopter quadrotor [D].University of Pennsylvania, 2003.

[9] Yu Y, SunFeng, Wang Y. Controller design of quadrotor aerial robot[J]. Physics Procedia, 33(2012) 1254-1260.

[10] Huanye Liu. Flight control system research and design of small four rotor aircraft [D]. Shanghai jiaotong university, 2009.

[11] Yang Y, Wu J, Zheng W. Variable structure attitude control for an UAV with parameter uncertainty and external disturbance[J]. Procedia Engineering, 15(2011)408-415. 